Delft University of Technology

\title{
State of the Art in Technology-Supported Resilience Training For Military Professionals
}

Favié, Joris; Ghazi Vakili, Vanessa; Brinkman, Willem-Paul; Morina, N; Neerincx, Mark

DOI

10.1515/9783110471137-013

Publication date

2016

Document Version

Final published version

Published in

Human Computer Confluence

\section{Citation (APA)}

Favié, J., Ghazi Vakili, V., Brinkman, W-P., Morina, N., \& Neerincx, M. (2016). State of the Art in

Technology-Supported Resilience Training For Military Professionals. In A. Gaggioli, A. Ferscha, G. Riva, S. Dunne, \& I. Viaud-Delmon (Eds.), Human Computer Confluence: Transforming Human Experience Through Symbiotic Technologies (pp. 229-240). Walter de Gruyter. https://doi.org/10.1515/9783110471137-013

\section{Important note}

To cite this publication, please use the final published version (if applicable).

Please check the document version above.

\section{Copyright}

Other than for strictly personal use, it is not permitted to download, forward or distribute the text or part of it, without the consent of the author(s) and/or copyright holder(s), unless the work is under an open content license such as Creative Commons.

Takedown policy

Please contact us and provide details if you believe this document breaches copyrights.

We will remove access to the work immediately and investigate your claim. 
Joris Favié, Vanessa Vakili, Willem-Paul Brinkman, Nexhmedin Morina and Mark A. Neerincx

\title{
13 State of the Art in Technology-Supported Resil- ience Training For Military Professionals
}

\begin{abstract}
Certain professions carry a risk of experiencing traumatic events and sometimes developing Posttraumatic Stress Disorder (PTSD). Researchers have been working on strategies to prevent professionals in those fields from developing PTSD. Recently, there has been a focus on applying technology that supports these preventive strategies, especially in a military context. This chapter provides an overview of the state of the art in technology-supported resilience training for military professionals, which has considerable common ground with the interdisciplinary field of human computer confluence (HCC). The effectiveness of these technologies and the resilience strategies they support are reviewed through the literature.
\end{abstract}

Keywords: Resilience, Training Systems, Posttraumatic Stress Disorder

\subsection{Introduction}

Soldiers, police officers, fire fighters, and ambulance staff are significantly more likely to experience traumatic events and other forms of intense stress at work. They might witness situations in which people are seriously injured or killed. They themselves might also be exposed to threatened or actual injury as well as threatened or actual death. Exposure to these and similar traumatic events can lead to posttraumatic stress disorder (PTSD). Individuals with PTSD re-experience the traumatic event via intrusive memories or nightmares, have increased physiological reactions upon exposure to reminders of the traumatic event, avoid distressing reminders of traumatic events, and have negative alterations in cognitions about themselves and the world as well as negative alterations in arousal and reactivity (American Psychiatric Association, 2013). Significant functional impairment has been shown in the daily lives of those meeting criteria for PTSD (Nemeroff et al., 2006). Extensive work has been done on the treatment of PTSD (Ehlers et al., 2010). Recently, research has also focused on ways to prevent PTSD by enhancing resilience before a trauma occurs with computer assisted training programs.

This work has considerable common ground with the HCC research agenda, which focuses on the science and technologies required to ensure an effective, transparent coexistence between humans and computers, and studies how to enable radically new forms of sensing, perception, interaction, and understanding. The HCC 
paradigm brings together fields such as bio-signals processing, neuroscience, electronics, robotics, pervasive computing, virtual \& augmented reality (Ferscha, 2013).

Various resilience training environments employ pervasive technologies to collect biosignals to give professionals real time insight into their response to stressful events simulated, for example, in a virtual reality environment (Bouchard, Bernier, Boivin, Morin, \& Robillard, 2012). To be effective, these technologies should not hinder the experience, but fade into the background of users' awareness. This can only be done with extensive understanding of the mental processes set in motion when an individual is confronted with traumatic events, and the psychology behind resilience training as well as resilience measurement. This chapter gives a coherent overview of these issues before discussing various resilience training-systems that have been developed recently across the world.

\subsection{Psychology}

PTSD symptoms have been explained as the result of an inability to inhibit learned fear in a safe environment. During a traumatic event, certain neutral stimuli become associated with fearful stimuli. After the event, the neutral stimuli continue to elicit the negative or fearful responses they have now been associated with. Social-cognitive theories like Horowitz's (1986) stress response theory explain how trauma breaks existing mental structures, and how incompatible information is reconciled with previous beliefs. Horowitz argued that when faced with trauma, two responses occur: (1) outcry at the realization of the trauma, and (2) an attempt to integrate the new trauma information with prior knowledge. Horowitz proposed that failure to process the trauma information in the second response leads to PTSD as the information remains in active memory and continues to intrude and be avoided. While social-cognitive theories focus on the wider personal and social context, cognitive information processing theories center mainly on the traumatic events itself (Creamer, Burgess, \& Pattison, 1992; Foa, Steketee, \& Rothbaum, 1989), or to be more precise on the encoding, storage, and recall of the traumatic events and their related stimuli and responses (Brewin \& Holmes, 2003). The core idea is that traumatic events are represented in memory in a special way, and if they are not processed and integrated within the broader memory system appropriately, psychopathology may result.

\subsubsection{Resilience}

Although many individuals develop PTSD after experiencing a traumatic event, most individuals exposed to a traumatic event show psychological resilience in the sense that they do not fall victim to a long term debilitating disorder, but manage to recover from the initial blow after some time (Mancini \& Bonanno, 2006). This has sparked 
research into the concept of resilience, which on the most basic level has been defined as the ability to adapt and cope successfully despite threatening or challenging situations (Bonanno, 2004). The empirical construct of resilience is however not that consistent, well defined and scientifically grounded yet. Resilience also is being perceived differently, as a set of characteristics, a trait, an outcome, a process or all of the above. Southwick and Charney (2012) describe resilience as the complex outcome of genetic, biological, psychological, social and spiritual factors. Resilience is often defined in terms of the presence of protective factors, which enables individuals to resist life stress (Kaplan et al., 1996). Rutter (1990) defined three general variables as protective factors: personality coherence, family cohesion, and social support. For personality factors he included level of autonomy, self-esteem and self-efficacy, good temperament, and positive social outlook.

\subsubsection{Hardiness}

Hardiness is a personality style introduced by Kobasa (1981), which is closely related to resilience. The construct of hardiness is posited as mediating stress and illness, potentially reducing the negative effects of stress. Theoretically, hardiness develops in early childhood and emerges as the result of rich, varied, and rewarding life experiences. Hardiness comprises three sub-constructs: (1) commitment as opposed to alienation, (2) control as opposed to powerlessness, and (3) challenge as opposed to threat. Individuals who are under stress will stay healthier if they feel committed to the various areas of their lives. Commitment is the valuing of one's life, one's self, one's relationships, and the investment of oneself in these valued dimensions of life. Commitment results in a sense of purpose that can carry a person through difficult times. Having a sense of control over what takes place in their lives helps individuals to understand stress and stay healthier than those who feel helpless in the face of external powers. People with control can integrate various kinds of events into a continuing life plan, and transform these events into something consistent with meaning, which makes them less destructive to their wellbeing. Persons with this view believe that change, rather than stability, is the norm in their life (Kobasa, 1982).

\subsection{Measuring Resilience}

Instead of trying to measure resilience directly, it is often measured indirectly as a function of other constructs (e.g. hardiness), clinical outcomes (e.g., absence of depression or PTSD), or behavioral measures (e.g. academic performance). Selfreport instruments are used widely in studies related to resilience training. There are a number of self-report measurement scales and instruments related with resilience to support research and clinical practice: 
- $\quad$ Baruth protective factors inventory (Baruth \& Carroll, 2002)

- Youth risk and resilience inventory (Brady, 2006)

- Connor-Davidson resilience scale (Connor \& Davidson, 2003)

- Resilience Scale for Adults (Friborg, et al., 2006)

- $\quad$ Resilience scale (Wagnild \& Young, 1993)

In addition, the Deployment Risk and Resilience Inventory (King, King \& Vogt et al., 2006) has been specially developed for military personnel.

\subsubsection{Biomarkers}

To improve objectivity, psychosocial instruments could be expanded to integrate biomarkers with demonstrated relevance to resilience. A biomarker is an objectively measured indicator of a biological state. Examples of biomarkers are hormones, genetic data and neurological factors. Biomarker research related to psychological performance and dysfunction is currently in the early stages. Rather than attempting to measure resilience itself, it is often measured using biomarkers that are associated with better-defined constructs such as stress or arousal, e.g. skin conductivity, heart rate, heart rate variability (HRV), and salivary cortisol.

Cortisol is a hormone released in response to stress. Skin conductance, also known as galvanic skin response (GSR), is a measure of the electric conductance of the skin, which varies with the level of sweat released in response to arousal. GSR has been found to be associated with self-reported sadness (Kreibig, Wilhelm, Roth, \& Gross, 2007) and writing about traumatic experiences (Hughes, Uhlmann, \& Pennebaker, 1994). HRV reflects the fluctuations in time between two consecutive heartbeats, which is considered to be an indication of the constant interaction of the sympathetic and parasympathetic circuits of the autonomous nervous system (ANS) innervating the heart (Taelman \& Vandeput, 2009). HRV therefore provides a means to quantify the activity of the ANS, and may thus provide a measure for stress. Although the causes of HRV are still under debate, it can be considered a general measure of adaptability as HRV reduces as people become more vulnerable to stress (Lehrer, 2007).

Preliminary genetic studies have identified some loci for resilience, but which specific genes confer resilience via specific mechanisms is still an active area of research. Epigenetic factors have also emerged as candidates for gene-environment interactions that influence stress responsiveness (Franklin, Saab \& Mansuy, 2012). Epigenetics is the study of heritable changes in gene expression without altering the DNA sequence itself, e.g. through DNA methylation: transcription of genes has found to be inhibited when methyl groups are attached to them. This process of DNA methylation may apparently be brought on by environmental factors: Meaney \& Szyf (2005) found that in the pups of inattentive mother rats, genes regulating the production of 
glucocorticoid receptors, which regulate sensitivity to stress hormones, were highly methylated. Furthermore, this methylated DNA was passed on to the next generation. As epigenetic measures are thought to reflect enduring effects of environmental exposures, they may be useful in distinguishing e.g. combat-exposed veterans who do or do not develop PTSD (Yehuda, Daskalakis, Desarnaud, et. al, 2013).

The construct of allostatic load is based on multiple biomarkers. Allostatic load is a measure of cumulative wear and tear on physiological symptoms due to chronic stress (McEwen \& Stellar, 1993) and is based on a response called allostasis.

\subsubsection{Emotional Stroop Test}

The Stroop effect demonstrates the presence of interference in the reaction time of a task (Stroop, 1935). When the name of a color is printed in a font color other than its name (e.g., the word red printed in blue instead of red), naming the color of the word takes longer than when the color of the font matches the name of the color. The emotional Stroop test (EST) is an adaptation of the original Stroop test, and measures an individual's ability to ignore irrelevant emotional content by examining the response time of the individual to name the colors of words that are emotionally charged for him or her. Instead of relying on mere lexical representations, images have also been used in a so-called Pictorial Emotional Stroop Task (PEST). In a PEST, images are either surrounded by a colored border, or they have been color-filtered so that they appear tinted in a single color. People are slower to name the color of a word or image that is emotionally charged for them, which is interpreted as a selective allocation of attentional processes to emotionally charged information (Williams, Mathews, MacLeod, 1996). This process takes a few milliseconds, and competes with other cognitive processes such as recognizing the color. The latter processes will thus be performed slower as compared to a situation lacking stimuli that are perceived as threatening. Research (MacLeod \& Hagan, 1992) using the EST indicates that preexisting differences in attention control can predict adjustment to stress. Furthermore, in a meta-analysis of 26 studies Cisler et al. (2011) conclude that EST performance of PTSD groups was affected by PTSD-relevant words and generally threatening words relative to neutral words, and the performance of trauma exposed control groups was impaired only by PTSD-relevant words. These effects were not present in the nontrauma exposed control groups.

\subsubsection{Startle Reflex}

The startle reflex is a natural physiological response to unexpected, intense stimuli such as a loud noise or a bright flash of light. It manifests itself by muscle responses such as contraction of the neck muscles, jumping up and blinking of the eyes, and 
serves to protect the back of the neck (whole-body startle) or the eye (eye blink). The reflex is influenced by emotional states. For normal individuals the amplitude of the reflex is larger when presented together with unpleasant stimuli (e.g. pictures of a mutilated body), smaller with neutral stimuli (e.g. a picture of a closet), and still smaller when presented with pleasant stimuli (e.g. a picture of a smiling baby). (Vrana, Spence, \& Lang, 1988). The amplitude is also larger when fear is elicited beforehand, which is referred to as the fear potentiated startle (FPS) (Brown, Kalish, \& Farber, 1951). A conditioned fear response can be generated by e.g. repeatedly shining a light or showing a colored object on a screen followed by an aversive stimulus such as an air-blast to the larynx or an electric shock. If a loud noise that triggers the startle response is now presented shortly after (or together with) the shining light, the startle response will be significantly larger. The FPS response also occurs after seeing videos or slides with unpleasant or anxiety-provoking scenes (Vrana et al., 1988). An increased FPS response is present in individuals with PTSD, and several studies found that the FPS becomes even bigger when they experience stress (Lissek et al., 2005). Furthermore, people diagnosed with PTSD display a similar FPS response to threatening and neutral stimuli, indicating that they cannot distinguish between a stimulus as a genuine threat or being harmless (Pole, Neylan, Best, Orr \& Marmar, 2003). Moreover, war veterans with severe, chronic PTSD have a significantly reduced ability for the extinction of conditioned fear responses (Milad et al., 2008).

\subsubsection{Content Analysis of Narratives}

There have been numerous studies on the beneficial effects of emotional writing. In a meta-analysis of 13 experimental writing studies, Smyth (1998) reported that personally disclosive writing was linked with improved physical and mental health. These positive effects have been explained by the idea that constructing a narrative helps individuals to better integrate the experience. Support for this idea is provided by studies showing that health improvements can be predicted by automated linguistic analysis, e.g. the number of emotion words people use may be indications about their emotional states, thought processes, motivations, and intentions (Tausczik \& Pennebaker, 2009). Examples of other predicting word patterns are (1) an increasing use of cognitive or insight words over the days of writing (Pennebaker, Mayne, \& Francis, 1997), (2) shifts in the use of pronouns from day to day (Campbell \& Pennebaker, 2003), (3) higher use of positive relative to negative emotion words (Pennebaker et al., 1997). 


\subsection{Resilience Training}

Concepts of cognitive behavior therapy (CBT) are used in applied training settings as discussed later on in this chapter. CBT is based on the assumption that cognitively distorted views of experiences and events may cause psychological problems. Furthermore, CBT theories presume that psychological problems are maintained by faulty thinking and behavior patterns. The basic cognitive behavior model in CBT states there is a two-way link between cognition, emotion and behavior in which cognitive processes can influence emotions and behavior, and behavioral change can influence cognitions and emotions (Mowrer \& Orville, 1950). Here, fear is seen as a response resulting from classical conditioning, and it is maintained and negatively reinforced by avoidance. A core idea is also that there is no direct link between an event and how people feel, but it is the way they perceive situations, i.e. cognitive appraisal, that influences their emotions. The core tenet of CBT is that psychological problems arise when there is a mismatch between the perception of events and the available evidence of reality gets exaggerated beyond the available facts. CBT techniques are used to help people challenge their automatic thoughts and schemas, and replace maladaptive thoughts with more sound and healthy thoughts, in order to decrease emotional distress and dysfunctional behavior (Hassett \& Gevirtz, 2009).

\subsubsection{Stress Inoculation Training (SIT)}

SIT (Meichenbaum \& Deffenbacher, 1988) is a form of CBT aimed at the reduction and prevention of stress. The idea is that arousal levels of individuals in response to powerful stressors may be reduced by inoculating them to potentially traumatizing stressors. It is based on models that posit that stress arises when the perceived demands of a situation exceed an individual's (perceived) capability to cope. SIT is designed to enable individuals to manage minor stressors in order to increase their ability to manage major stressors, and to boost their confidence in use of their coping skills. Meichenbaum proposes three stages to SIT: (1) psycho-education (learning about stress responses and the need to control them), (2) training (learning a skill, such as arousal control, in order to tone down the harmful effects of stress), and (3) implementation (utilizing these skills in the stressful context).

\subsubsection{Biofeedback Training}

Biofeedback is a method used for teaching voluntary control of various physiological functions in order to improve health or performance by providing perceptible, realtime feedback for variations in physiological activity (Schwartz \& Andrasik, 2003). Feedback is usually given in the form of visual and/or auditory signals derived from 
physiological recording devices. This provides both the trainee and the trainer with a continuous image of what is going on inside the body. The effect of a frightening thought, the way of breathing etc. is directly visible. There are three stages of biofeedback training: (1) acquiring awareness of the maladaptive response, here individuals learn the effect of certain thoughts and bodily events on a response, (2) learning to control the response, and (3) transfer these control skills into everyday life (Calderon \& Thompson, 2004). In treatment of stress-related disorders, biofeedback is often combined with relaxation training, which is referred to as biofeedback-assisted relaxation training.

\subsubsection{Cognitive Reappraisal}

According to appraisal theories of emotion, emotion is determined by an individual's subjective appraisal of an event - the meaning and significance that the individual attaches to the event - rather than the event itself (Lazarus \& Folkman, 1984). Therefore, learning to change one's own appraisal process is believed to be a key factor in many psychological interventions such as CBT (Samoilov \& Goldfield, 2000). A cognitive-linguistic emotion regulation strategy known as cognitive reappraisal aims to achieve exactly this (Gross, 1998). Cognitive reappraisal refers to the implementation of cognitive strategies to assist individuals in changing the intensity of their response to an event (Ochsner \& Gross, 2005). In reappraisal tasks, trainees may be asked to systematically apply certain appraisal themes so as to reduce the negative affect they experienced. Schartau, Dalgleish, \& Dunn (2009) found that many of the dysfunctional appraisal themes mentioned in the CBT literature reflect a difficulty to see the bigger picture. They selected four functional appraisal themes focused on perspective broadening, e.g. a theme that stresses that bad events do not happen often and good things occur all the time. In their study, they asked participants to watch a film and employ these appraisal themes to reduce their emotional reaction. Participants who practiced appraisal displayed reduced levels of self-reported negative emotional responses to a final distressing test film. Furthermore, appraisal practice resulted in reduced intrusion and avoidance of the target memories in the week after the study.

Several other laboratory studies (e.g. Dandoy \& Goldstein, 1990) also found that people instructed to use reappraisal reported less negative emotion after watching an emotional film clip compared to those in a just watch condition. Moreover, Gross \& John (2003) reported that individuals who frequently use reappraisal, experience less negative emotions in emotionally charged events and exhibit positive outcomes over time. 


\subsection{Review of Resilience Training Systems}

The focus on HCC in the mental healthcare domain is not new as applications such as brain interfacing, mobile devices, body computing, and virtual reality have been studied in this context (Viaud-Delmon, Gaggioli, Ferscha, et al., 2012). The same seems to apply for the current resilience training systems as described below.

\subsubsection{Predeployment Stress Inoculation Training (Presit) \& Multimedia Stressor Environment (Mse)}

Hourani, Kizakevech, et al. (2011) developed a SIT based intervention as a preventative effort against combat related stress disorders in armed forces personnel. The main goal of this approach is to train personnel to lower their physiologic arousal in response to a stressor. A group-administered program was chosen because it was cost-effective, and because marines are used to working in groups where they can also support each other. The skills acquisition phase of the training is a presentation of two types of breathing and focus-retraining techniques. The eyes-open technique focuses on in-combat situations, while the eyes-closed technique focuses on postcombat situations. Trainees are also trained in stress reduction using a biofeedback training software package that provides paced breathing exercises via a graphical user interface, including display of HRV and a stress index. The training is practiced in a combat relevant MSE. Up to 20 individuals are seated in front of a large screen, and they are taken on a virtual journey through an Iraqi village from a first person perspective, as if they are all in the same multi-passenger vehicle. Trainees are instructed to perform a reaction time task by pressing specific buttons upon noticing specific events. A controlled pilot study was carried out to compare the efficacy of PRESIT with current best practices of the Navy's and Marine's Combat and Operational Stress Control programs by examining HRV and reaction time in response to events in the MSE. Participants with deployment experience, especially those with PTSD, showed greater relaxation during the MSE of a follow-up session relative to that of a baseline session. There was no significant effect for individuals without combat experience.

\subsubsection{Stress Resilience in Virtual Environments (Strive)}

In contrast to the narrow focus of the PRESIT study on lowering physiologic arousal in response to a stressor through breathing and focus re-training, STRIVE aims for a broader CBT based approach. STRIVE is a set of combat simulations that are part of a multi-episode free-agency narrative experience, forming an experiential learning approach for delivering psycho-educational material, stress management techniques and cognitive-behavioral emotional coping strategies prior to deployment 
(Rizzo et al., 2011). This approach draws input from CBT and teaches soldiers the distinction between factual events and emotion caused by their appraisal that can be taught. STRIVE is designed as a virtual emotional obstacle course, including various cinematic VR episodes that are narrated in the first-person as if by the individual experiencing the training. At the end of each episode an emotionally challenging event occurs, and a virtual mentor appears, who guides the soldiers through rational restructuring exercises relevant to the event. This facilitates state-dependent learning as skills are taught in a similar state in which they have to be applied later on. STRIVE uses cinematic techniques, such as a storyline and character development, so that soldiers can become engaged on an emotional level.

\subsubsection{Immersion and Practice of Arousal Control Training (Impact)}

Bouchard et al (2012) developed a stress management training (SMT) program to test whether practicing stress management skills would benefit the usual training offered to military personnel. A stressful 3D horror/first person shooting game was modified by making it more stressful and including biofeedback components, so that it could be used as an SIT based training tool for biofeedback-assisted SMT. Visual biofeedback was provided by reducing the visual field of the player more and more as arousal (as measured by skin conductivity and heart rate variability) of the participant increased. Auditory feedback was provided by the sound of a thumping heart that grew progressively louder and faster. Trainees play the game during three 30-minute training sessions, and a human coach is present to personally help train participants in tactical breathing during these sessions. The program was evaluated by measuring salivary cortisol and heart rate during a live first-aid training simulation after soldiers participated in the ImPACT program. A significant reduction in stress level was found for the soldiers that followed the program as compared with training as usual.

\subsubsection{Personal Health Intervention Tool (PHIT) for Duty}

Kizakevich, Hubal and Brown (2012) developed a personal health assessment and self-help intervention application for smartphones and tablets to help build resilience in healthy military personnel and support prevention in high-risk personnel. The application collects a host of health assessments using self-report forms and questionnaires. In addition data are acquired via interactive exercises and optional nonintrusive physiological and behavioral sensors measuring e.g. HRV and body motion. An intelligent advisor analyzes these assessments using a personal health status model to recommend, tailor and present self-help advisories to reduce symptoms and prevent disease, such as stress relaxation, mindfulness meditation, progressive muscle relaxation, behavior therapy, and links to professional care. 


\subsubsection{Stress Resilience Training System}

Cohn, Weltman, Ratwani et al (2010) developed an application that combines cognitive learning methodologies grounded in learning theory and biofeedback techniques. It is based on the idea that fighters adapt to combat stress after the first few experiences by learning self-awareness of their stress state and self-regulation of stress effects, and the idea that training can help duplicate this process. The application consists of three parts: (1) know-how, (2) coherence training, and (3) HRV-controlled games, which roughly correspond to the three stages of SIT. The know-how part consists of brief audiovisual presentations. The main goal is to provide the trainee with techniques to inoculate against negative stress effects and use positive effects of stress. The coherence training includes two elements. A HRV-controlled game allows trainees to practice and master a coherence breathing technique. To practice their technique the trainees are presented with games with ascending levels of difficulty. A virtual coach advises the trainees during the training.

\subsubsection{Physiology-Driven Adaptive Virtual Reality Stimulation for Prevention and Treatment of Stress Related Disorders}

Cosic, Popovic, Kukolja et al. (2010) describe a concept for an adaptive control of virtual reality affect stimulation. Exposure levels are automatically controlled according to physiological input levels, which would alleviate trainers from performing this task. The three main components are: (1) stimulus generator that contains various rated and categorized multimedia stimuli, (2) emotional state estimator, which collects physiological samples from trainees in order to estimate their valence/arousal, and (3) adaptive controller, which applies a strategy for the control of the stimulus generator based on this estimation. A stimulus-delivery algorithm is also described that automatically adjusts for the participants' habituation, by choosing for stimuli that are either more or less intense.

\subsection{Conclusions}

Concepts from CBT are found to be widely used, and both SIT and biofeedback training have been implemented within technology-supported resilience trainings. Cognitive reappraisal has also been implemented in one existing training system. The preferred approach to measure resilience currently seems to combine subjective selfreport with objective measures such as biomarkers, of which GSR and HRV are often used. Most of the current systems involve breathing and relaxation techniques as a means to reduce stress or arousal during stressful activities. Unfortunately, limited empirical evidence is currently available regarding the efficacy of these systems in 
helping individuals staying more relaxed during stressful exercises, which often made it only possible for this chapter to describe the systems and the psychology behind them, and not their efficacy. A major limitation of the current programs is also the lack of longitudinal evaluation. At this point no conclusion can be made on the long-term efficacy of these training systems. Existing systems still need further empirical evaluation and new technology-supported training paradigms need to be explored. Although some elements of HCC are already being employed, integrating a more full set of interdisciplinary HCC components into an effective resilience training tool remains both a challenge and an opportunity.

Acknowledgement: This study was supported by the Dutch Economic Structure Enhancement Fund (FES) "Brain and Cognition: Societal Innovation" program (NWO project no 056-25-012).

\section{References}

American Psychiatric Association (2013). Diagnostic and statistical manual of mental disorders, 5th edition. Arlington: American Psychiatric Association.

Baruth, K. E., \& Caroll, J. J. (2002). A formal assessment of resilience: The Baruth Protective Factors Inventory. The Journal of Individual Psychology; The Journal of Individual Psychology.

Bonanno, G. A. (2004). Loss, trauma, and human resilience: have we underestimated the human capacity to thrive after extremely aversive events? The American psychologist, 59(1), 20-28.

Bouchard, S., Bernier, F., Boivin, E., Morin, B., \& Robillard, G. (2012). Using biofeedback while immersed in a stressful videogame increases the effectiveness of stress management skills in soldiers. PloS one, 7(4), e36169.

Brady, R. P. (2006). Youth Risk and Resilience Inventory. JIST Publishing.

Brewin, C. R., \& Holmes, E. A. (2003). Psychological theories of posttraumatic stress disorder. Clinical Psychology Review, 23(3), 339-376.

Brown, J. S., Kalish, H. I., \& Farber, I. E. (1951). Conditioned fear as revealed by magnitude of startle response to an auditory stimulus. Journal of Experimental Psychology, 41(5), 317.

Calderon, K. S., \& Thompson, W. W. (2004). Biofeedback relaxation training: A rediscovered mind-body tool in public health. American Journal of Health Studies, 19(4), 185.

Campbell, R. S., \& Pennebaker, J. W. (2003). Research Article The Secret Life Of Pronouns : Flexibility in Writing Style and Physical Health. Psychological Science, 14(1), 60-65.

Cisler, J. M., Wolitzky-Taylor, K. B., Adams, T. G., Babson, K. A., Badour, C. L., \& Willems, J. L. (2011). The emotional Stroop task and posttraumatic stress disorder: a meta-analysis. Clinical psychology review, 31(5), 817-828.

Cohn, J., Weltman, G., Ratwani, R., Chartrand, D., \& McCraty, R. (2010). Stress Inoculation through Cognitive and Biofeedback Training. The Interservice/Industry Training, Simulation \& Education Conference (I/ITSEC) (Vol. 2010, pp. 1-11).

Connor, K. M., \& Davidson, J. R. T. (2003). Development of a new resilience scale: The ConnorDavidson resilience scale (CD-RISC). Depression and anxiety, 18(2), 76-82.

Cosić, K., Popović, S., Kukolja, D., Horvat, M., \& Dropuljić, B. (2010). Physiology-driven adaptive virtual reality stimulation for prevention and treatment of stress related disorders. Cyberpsychology, behavior and social networking, 13(1), 73-78. 
Creamer, M., Burgess, P., \& Pattison, P. (1992). Reaction to trauma: a cognitive processing model. Journal of Abnormal Psychology, 101(3), 452-459.

Dandoy, A. C., \& Goldstein, A. G. (1990). The use of cognitive appraisal to reduce stress reactions: A replication. Journal of Social Behavior \& Personality; Journal of Social Behavior \& Personality.

Driskell, J. E., \& Johnston, J. H. (1998). Stress exposure training. Making decisions under stress: Implications for individual and team training, 191-217.

Ehlers, A., Bisson, J., Clark, D. M., Creamer, M., Pilling, S., Richards, D., Yule, W. (2010). Do all psychological treatments really work the same in posttraumatic stress disorder? Clinical psychology review, 30(2), 269-276.

Ferscha, A. (Ed.). (2013). Human computer confluence, The Next Generation Humans and Computers Research Agenda: Institute for Pervasive Computing, Johannes Kepler University Linz.

Foa, E. B., Steketee, G., \& Rothbaum, B. O. (1989). Behavioral/cognitive conceptualizations of post-traumatic stress disorder. Behavior Therapy, 20(2), 155-176.

Franklin, T. B., Saab, B. J., \& Mansuy, I. M. (2012). Neural mechanisms of stress resilience and vulnerability. Neuron, 75(5), 747-761

Friborg, O., Hjemdal, O., Rosenvinge, J. H., \& Martinussen, M. (2006). A new rating scale for adult resilience: What are the central protective resources behind healthy adjustment? International journal of methods in psychiatric research, 12(2), 65-76.

Gross, J. J. (1998). The emerging field of emotion regulation: An integrative review. Review of general psychology, 2(3), 271.

Gross, J. J., \& John, O. P. (2003). Individual differences in two emotion regulation processes: implications for affect, relationships, and well-being. Journal of personality and social psychology, 85(2), 348-362.

Hassett, A. L., \& Gevirtz, R. N. (2009). Nonpharmacologic treatment for fibromyalgia: patient education, cognitive-behavioral therapy, relaxation techniques, and complementary and alternative medicine. Rheumatic diseases clinics of North America, 35(2), 393.

Horowitz, M. J. (1986). Stress-response syndromes: A review of posttraumatic and adjustment disorders. Hospital \& Community Psychiatry, 37(3), 241-249.

Hourani, L., Kizakevich, P., \& Hubal, R. (2011). Predeployment stress inoculation training for primary prevention of combat-related stress disorders. Journal of cybertherapy \& rehabilitation, 4(1).

Hughes, C. F., Uhlmann, C., \& Pennebaker, J. W. (1994). The Body's Response to Processing Emotional Trauma: Linking Verbal Text with Autonomic Activity. Journal of Personality, 62(4), 565-585.

Ilnicki, S., Wiederhold, B. K., Maciolek, J., \& Kosinska, L. (2012). Effectiveness Evaluation for Short-Term Group Pre-Deployment VR Computer- Assisted Stress Inoculation Training Provided to Polish ISAF Soldiers. In B. K. Wiederhold \& G. Riva (Eds.), Annual Review of Cybertherapy and Telemedicine (pp. 113-117). IOS Press.

Kaplan, C. P., Turner, S., Norman, E., \& Stillson, K. (1996). Promoting resilience strategies: A modified consultation model. Children \& Schools, 18(3), 158-168.

King, L. A., King, D. W., Vogt, D. S., Knight, J., \& Samper, R. E. (2006). Deployment Risk and Resilience Inventory: A collection of measures for studying deployment-related experiences of military personnel and veterans. Military Psychology, 18(2), 89-120.

Kizakevich, P., Hubal, R., \& Brown, J. (2012). PHIT for Duty, a Mobile Approach for Psychological Health Intervention. Studies in health technology and informatics, 268-272.

Kobasa, Suzanne C, Maddi, S. R., \& Courington, S. (1981). Personality and Constitution as Mediators in the Stress-Illness Relationship. Journal of Health and Social Behavior, 22(4), pp. 368-378.

Kobasa, Suzanne C, \& others. (1982). The hardy personality: Toward a social psychology of stress and health. Social psychology of health and illness, 4, 3-32. 
Kreibig, S. D., Wilhelm, F. H., Roth, W. T., \& Gross, J. J. (2007). Cardiovascular, electrodermal, and respiratory response patterns to fear- and sadness-inducing films. Psychophysiology, 44(5), 787-806.

Lazarus, R. S., \& Folkman, S. (1984). Stress, appraisal, and coping. Springer Publishing Company.

Lehrer, P. M. (2007). Biofeedback training to increase heart rate variability. Principles and practice of stress management, 227-248.

Lissek, S., Powers, A. S., McClure, E. B., Phelps, E. A., Woldehawariat, G., Grillon, C., \& Pine, D. S. (2005). Classical fear conditioning in the anxiety disorders: a meta-analysis. Behaviour research and therapy, 43(11), 1391-1424.

MacLeod, C., \& Hagan, R. (1992). Individual difference in the selective processing of threatening information, and emotional responses to a stressful life event. Behaviour Research and Therapy, 30(2), 151-161.

Mancini, A. D., \& Bonanno, G. A. (2006). Resilience in the face of potential trauma: clinical practices and illustrations. Journal of clinical psychology, 62(8), 971-985.

McEwen, B. S., \& Stellar, E. (1993). Stress and the individual: mechanisms leading to disease. Archives of internal medicine, 153(18), 2093.

Meaney, M., \& Szyf, M. (2005). Environmental programming of stress responses through DNA methylation: life at the interface between a dynamic environment and a fixed genome. Dialogues in Clinical Neuroscience, 3, 103-123.

Meichenbaum, D. H., \& Deffenbacher, J. L. (1988). Stress inoculation training. The Counseling Psychologist, 16(1), 69-90.

Milad, M. R., Orr, S. P., Lasko, N. B., Chang, Y., Rauch, S. L., \& Pitman, R. K. (2008). Presence and acquired origin of reduced recall for fear extinction in PTSD: results of a twin study. Journal of psychiatric research, 42(7), 515-520.

Mowrer, O. (1950). Learning theory and personality dynamics: selected papers. New York: Ronald Press.

Nemeroff, C. B., Bremner, J. D., Foa, E. B., Mayberg, H. S., North, C. S., \& Stein, M. B. (2006). Posttraumatic stress disorder: a state-of-the-science review. Journal of psychiatric research, 40(1), 1-21.

Ochsner, K. N., \& Gross, J. J. (2005). The cognitive control of emotion. Trends in cognitive sciences, 9(5), 242-249.

Pennebaker, J., Mayne, T. J., \& Francis, M. E. (1997). Linguistic predictors of adaptive bereavement. Journal of personality and social psychology, 72(4), 863-871.

Pole, N., Neylan, T. C., Best, S. R., Orr, S. P., \& Marmar, C. R. (2003). Fear-potentiated startle and posttraumatic stress symptoms in urban police officers. Journal of Traumatic Stress, 16(5), 471-479.

Rizzo, A., Parsons, T. D., Lange, B., Kenny, P., Buckwalter, J. G., Rothbaum, B., Difede, J., et al. (2011). Virtual reality goes to war: a brief review of the future of military behavioral healthcare. Journal of clinical psychology in medical settings, 18(2), 176-187.

Rutter, M. (1990). Psychosocial resilience and protective mechanisms. In J. Rolf, A. S. Masten, D. Cicchetti, K. H. Neuchterlein, \& S. Weintraub (Eds.), Risk and protective factors in the development of psychopathology (pp. 181-214). New York: Cambridge University Press.

Samoilov, A., \& Goldfried, M. R. (2000). Role of Emotion in Cognitive-Behavior Therapy. Clinical Psychology: Science and Practice, 7(4), 373-385.

Schartau, P. E. S., Dalgleish, T., \& Dunn, B. D. (2009). Seeing the bigger picture: training in perspective broadening reduces self-reported affect and psychophysiological response to distressing films and autobiographical memories. Journal of abnormal psychology, 118(1), 15.

Schwartz, M. S., \& Andrasik, F. (2003). Biofeedback: A practitioner's guide. Guilford Press.

Smyth, J. M. (1998). Written emotional expression: effect sizes, outcome types, and moderating variables. Journal of consulting and clinical psychology, 66(1), 174-184. 
Southwick, S. M., \& Charney, D. S. (2012). Resilience: The Science of Mastering Life's Greatest Challenges (p. 204). Cambridge University Press.

Stroop, J. R. (1935). Studies of interference in serial verbal reactions. Journal of experimental psychology, 18(6), 643.

Taelman, J., \& Vandeput, S. (2009). Influence of mental stress on heart rate and heart rate variability. IFMBE Proceedings, 22, 1366-1369.

Tausczik, Y. R., \& Pennebaker, J. W. (2009). The Psychological Meaning of Words: LIWC and Computerized Text Analysis Methods. Journal of Language and Social Psychology, 29(1), 24-54.

Viaud-Delmon, I., Gaggioli, A., Ferscha, A., \& Dunne, S. (2012). Human Computer Confluence Applied in Healthcare and Rehabilitation. Studies in health technology and informatics, 181(Annual Review of Cybertherapy and Telemedicine), 42-45.

Vrana, S. R., Spence, E. L., \& Lang, P. J. (1988). The startle probe response: A new measure of emotion? Journal of abnormal psychology, 97(4), 487.

Wagnild, G. M., \& Young, H. M. (1993). Development and psychometric evaluation of the Resilience Scale. Journal of Nursing Measurement, 1(2), 165-178

Williams, J. M. G., Mathews, A., \& MacLeod, C. (1996). The emotional Stroop task and psychopathology. Psychological bulletin, 120(1), 3-24.

Yehuda, R., Daskalakis, N. P., Desarnaud, F., Makotkine, I., Lehrner, A. L., Koch, E., ... Bierer, L. M. (2013). Epigenetic Biomarkers as Predictors and Correlates of Symptom Improvement Following Psychotherapy in Combat Veterans with PTSD. Frontiers in Psychiatry, 4(September), 118. 